\title{
Analysis of Ethics for Using Portable Intelligent Devices based on PLS Regression Model
}

\author{
Xuecai Bao ${ }^{1}$, Jinghai Yin ${ }^{2}$, Tangsheng Wang ${ }^{2}$ \\ 1. School of Information Engineering, Nanchang Institute of Technology, Nanchang 330099, China \\ 2. Institute of Information technology, Jiangxi University of Technology, Nanchang 330098, China
}

Keywords: Analysis of ethics; Portable intelligent devices; Partial least-squares regression model

\begin{abstract}
With the development of wearable device, ethical issues widely attracted the attention of many people. The operating principles of technology and the basic definition of human well-being need to analyze the ethics issue for using portable intelligent devices. In this paper, the factors of impacting human healthy are described and the 21 variables are used for ethics analysis. Then, the partial least-squares regression model is proposed for discriminant analysis of ethics. The model between human healthy and portable intelligent equipment is established. Lastly, the result shows that the proposed method has higher prediction accuracy and the proposed contribution degree is valid for the ethics analysis.
\end{abstract}

\section{Introduction}

Since 2013, after Google glasses appear, many wearable devices are successively developed, such as navigation bracelet, golf gloves, senses railway, medical gloves recorder, children' intelligence pajamas, brainwave therapeutic apparatus, other reading device, the jet lag syndrome and so on. So-called wearable devices (wearable device) are directly wore in the body or integrated into the user's clothes or accessories of a portable device. Portable intelligent devices is not only a kind of hardware equipment, but also achieve powerful function through software support, data interaction and the cloud interaction and it will make a big change for our brain perceives and behavioral decision. Recently, domestic portable blood pressure meter, positioning bracelets, smart watches and other intelligent children also are developed.

In terms of device function, the development of the portable intelligent equipment services mainly includes six aspects: the health monitoring and consulting, the development of sensory abilities, tool life housekeeper, entertainment, social assistant, security guards. Like previous scientific and technological progress about the matter of human well-being in the history, the development of portable intelligent devices and applications is in favor of mankind at the same time. It will cause a series of ethical and moral confusion problems.

Greene et al[1] utilizes the FMRI technology to spy out brain and find out that the decisions of human in moral dilemmas are more associated with human emotional experience, rather than Kant's claim of rational [2]. With the emergence of a series of brain signal analysis technology, people predict that the neuroscience will realize the rapid development in the 21 st century. Recently, the Dutch scientists successfully use electric shock therapy to delete the specified memory in the human brain, even some scholars predict that human society will enter a "read brain age" [3]. The miniaturization of Hardware, the progress of brain computer interface technology and the coming of the big data era promote the development of nerve portable intelligent devices, which will become the future trend of intelligent equipment.

As for the aspects of practical application, this study will actively practice and promote activities of positive psychology [4] and advocate people should pursue happy life. Science first should be concerned with the fundamental of the human welfare, but the development of many modern technologies run counter to the goal of human life, which mainly includes that people makes efforts to cope with the continuous renewal of information and technology. Meantime, learning has lost its original fun and society has no enough time and space to help teenagers gradually to become 
independent. This study focuses on the discussion of neural ethical problems from using wearable technology. We opposed conformism stalled in the face of new technology and technology one-sidedly based on the foreign object. Meanwhile, for providing happiness of portable intelligent equipment for human being, we strive to overcome the dissimilation phenomenon brought by the portable intelligent equipment. In terms of negative aspects, with the advance of technology, human who lack sense of security reduce anxiety by satisfying physiological need. The portable intelligent device and read brain technology threatens people's privacy, and the development of brain-computer interface technology has swallowed the freedom of human being. Moreover, self-fulfilling space was squeezed by losing dignity, then all human leisure activities of restoring energy are controlled by portable intelligent equipment technology. Lastly, people even hope that technology itself can monitor and prevent brain addictive behavior [5]. For its positive effects, the cognitive neuroscientist of China, Bian Yufang, thought from the perspective of brain science, learning efficiency is better in relaxing mood. Furthermore, she said that early arranging to participate in all kinds of training classes will be harmful to children. The highest realm of education is self-education. The difference of children physical level cause different learning style preferences and the self-esteem may affect the person's life. The parents and teachers are the most important person in students' growth and development [6]. So, stop and isolation of new technology is not beneficial for the growth of the students.

In order to find a model between human healthy and portable intelligent equipment, the Partial Least Square Regression is proposed to use for exploring analysis model of ethics about portable intelligent equipment. This method is different from the traditional statistic method, which supplies researchers an applied way and is much simpler than neural network.

\section{Description of PLS Regression Model}

The basic ideal of PLS regression is to predict a set of dependent variables from a (very) large set of independent variables. In multiple linear regression models, there is a group of independent variables $Y=\left[\mathrm{y}_{1}, \ldots, \mathrm{y}_{q}\right]$ and a set of dependent variables $X=\left[x_{1}, \ldots ., x_{q}\right]$. When $q=1$, it is defined as a single dependent variable partial least-squares regression model (recorded as PLS1); if q>1, it is defined as more variable partial least-squares regression model. When all data meet Gauss -Markov assumptions, using least squares method, the predictor variables is

$$
\hat{Y}=X(\hat{X} X)^{-1} X^{\prime} Y
$$

$\hat{Y}$ is a good estimation of $Y$. From above formula, the matrix $\left(X^{\prime} X\right)$ must be reversible, so, when there are serious correlations in dependent variables $X$ or the number of sample points is less than the number of variables, the estimation of least square will be failure and trigger a series of difficulties in applications [7][8].

The specific descriptions are as follows:

The data matrix $E_{0}$ is the result of Normalization processing for $\mathrm{X}$ and the $F_{0}$ is a variable after normalization processing of $Y$. The specific processing is shown as formula (1) and (2).

$$
\begin{gathered}
F_{0 i}=\frac{y_{i}-\bar{y}}{s_{y}} \quad i=1,2,3, \ldots, \mathrm{n} \\
E_{0 i}=\frac{x_{i}-\bar{x}}{s_{x_{i}}} \quad i=1,2,3, \ldots, \mathrm{m}
\end{gathered}
$$

We define $t_{1}$ as the first factor of $E_{0} . w_{1}$ is the first eigenvector which is a unit vector, that is, $\left\|w_{1}\right\|=1$. Meanwhile, the $u_{1}$ is the first factor of $F_{0}, u_{1}=F_{0} c_{1}$. The $c_{1}$ is the first eigenvector of $F_{0}$ and $\left\|c_{1}\right\|=1$. On one hand, if $t_{1}$ and $u_{1}$ are requested greatly to represent the variability of $X$ and $Y$, according to the principle of principal component analysis, the $\operatorname{var}\left(u_{1}\right) \rightarrow \max$ and $\operatorname{var}\left(t_{1}\right) \rightarrow \max$. 
On the other hand, due to the need of regression model, the correlation of $t_{1}$ and $u_{1}$ is requested to get the maximum value, that is, $r\left(t_{1}, u_{1}\right) \rightarrow \max$.

So, the optimization problem is defined as below.

$$
\begin{aligned}
& \max _{w_{1} c_{1}}\left\langle E_{0} w_{1}, F_{0} c_{1}\right\rangle \\
& \text { s.t }\left\{\begin{array}{l}
w_{1}^{\prime} w_{1}=1 \\
c_{1}^{\prime} c_{1}=1
\end{array}\right.
\end{aligned}
$$

From formula (3), we know that the objective of this problem is mainly to maximize the $w_{1}^{\prime} E_{0}^{\prime} F_{0} c_{1}$ under the constraint condition of $\left\|w_{1}\right\|^{2}=1$ and $\left\|c_{1}\right\|^{2}=1$.

Here, we utilize the Lagrange algorithm, we can obtain:

$$
s=\dot{\psi} \dot{E}_{0} F_{0} \& \lambda\left({ }_{1} \dot{w} w_{1}-u_{1}\right) \lambda \quad\left({ }_{2}-\epsilon_{1} c\right.
$$

We can derivate the following equation.

$$
\begin{aligned}
& E_{0}^{\prime} F_{0} \dot{F}_{0} E_{0} \uplus_{1} \theta^{2} \\
& F_{0}^{\prime} E_{0}^{\prime} E_{0} F_{0} z_{1} \theta^{2}
\end{aligned}
$$

Where $w_{1}$ is eigenvector of matrix $E_{0}^{\prime} F_{0} F_{0}^{\prime} E_{0} w_{1}$ and the $\theta_{1}^{2}$ is eigenvalue. The $\theta_{1}$ is the value of objective function and it is required to get the maximum value. So, the $w_{1}$ is unit eigenvector of maximum eigenvector for matrix $E_{0}^{\prime} F_{0} F_{0}^{\prime} E_{0}$. Correspondingly, the $c_{1}$ is unit eigenvector of eigenvector $\theta_{1}^{2}$ for matrix $F_{0}^{\prime} E_{0}^{\prime} E_{0} F_{0}$. When the eigenvector $w_{1}$ and $c_{1}$ are solved, we can the factor extraction:

$$
\begin{gathered}
t_{1}=E_{0} n \\
u_{1}=F_{0} c
\end{gathered}
$$

Then, the two regression equation for $E_{0}$ and $F_{0}$ about $t_{1}$ are

$$
\begin{gathered}
E_{0}=t_{1} \dot{p}_{1}^{+} \quad \dot{1} \\
F_{0}=t_{1}^{\prime} r_{1}^{+},
\end{gathered}
$$

Where $p_{1}=E_{0}^{\prime} t_{1} /\left\|t_{1}\right\|^{2}, \quad r_{1}=F_{0}^{\prime} t_{1} /\left\|t_{1}\right\|^{2}$ denote respectively regression coefficient $E_{1}$ and $F_{1}$ residual matrix of regression equation. Then $E_{1}$ and $F_{1}$ instead of $E_{0}$ and $F_{0}$. Similarly, the value of $w_{1}, c_{1}, t_{2}$ and $u_{2}$ are computed by the repeating formula (7) and (8). When the $t_{h}$ is obtained, we stop the computation. The value of $h$ is determined by Cross-validation.

Finally, the partial least-squares regression equation for $F_{0}$ are obtained:

$$
\widehat{F}_{0}=r E y^{*}+r E_{1} w_{0}^{*}+{ }_{1 .}+r_{h} E w_{h}
$$

When the $y^{*}=\widehat{F}_{0}, x^{*}=E_{0 i}, a_{i}=\sum_{k=1}^{h} r_{k} w_{k i}^{*}$, the standard variable regression equation is defined.

$$
\widehat{y}^{*}=a_{1} x_{1}^{*}+a x \underset{2}{*} \frac{*}{2} \ldots+a_{m} x_{m}
$$




\section{The Analysis of Ethics for Using Portable Intelligent Devices}

Data Description. Jiangxi University of Technology and Nanchang Institute of Technology have more than 60,000 full-time students, from 30 provinces, municipalities, and autonomous regions and representing 19 ethnic minorities. This research investigated 2210 students proportionally from various departments and got 1936 effective cases, in which there are 1284 male students and 652 female students.

In this paper, 21 variables are arranged in the ethics Questionnaire for using portable intelligent devices. The 21 variables include health services, development, life convenience, entertainment, social and security, sensibility, memory, discrimination, description ability, fatigue, position affection, sight, smell, hearing, taste, touch, skin burning and tingling, nausea, headache, insomnia, loss of memory and privacy.

This study utilize neural science technology to analyze the social psychology effect brought by portable intelligent devices and focus on new technology of human mental health, education, training , happiness challenge and opportunity. Based on the analysis, we try to find out the solution for solving the crisis of the new technology ethics. In ethics questionnaire of portable intelligent devices, the 1136 sample data are used for clustering analysis based on two factors, namely, physiological aspects, and psychological aspects. The results showed that the ratio of sample categories is similar and center values of clustering remain unchanged.

Where class "1" represents focusing on the value of physiological aspects and class 2 represents the value of psychological aspects (see table 1). With samples of different numbers as a whole, the value of a single sample in respective type shows a strong stability (see table 2).

TABLE I NUMBER OF CASES IN EACH CLASS

\begin{tabular}{|l|l|l|l|l|l|l|l|l|l|l|l|}
\hline $\begin{array}{l}\text { Variabl } \\
\text { es }\end{array}$ & Si. & Sm. & He. & Ta. & Sb.t. & Na & Hea. & $\begin{array}{l}\text { In.1 } \\
\mathrm{m}\end{array}$ & Me. & To. & \\
\hline Class 1 & 4.12 & 4.23 & 3.22 & 4.5 & 4.2 & 4.1 & 4.7 & 3.1 & 3.7 & 4.3 & \\
\hline $\begin{array}{l}\text { Variabl } \\
\text { es }\end{array}$ & En. & Pr. & Se. & De. & Se, & $\begin{array}{l}\text { So.s } \\
\text { e }\end{array}$ & Fa. & P.a. & L.c. & D.a & Di. \\
\hline Class 2 & 4.1 & 5.1 & 4.9 & 3.1 & 4.5 & 2.1 & 4.8 & 3.1 & 2.3 & 3.2 & 2.2 \\
\hline
\end{tabular}

TABLE II FINAL CLASS CENTERS

\begin{tabular}{|c|c|}
\hline Class 1 & 532 \\
\hline Class 2 & 644 \\
\hline Valid & 1291 \\
\hline Missing & 29 \\
\hline
\end{tabular}

From Table 1 and Table 2, effective class achieves the possibility of establishing a distinction between the standards of individual types and provides discriminant analysis. In this paper, the class value of each object is made as the dependent variable, and the PLS regression model is proposed to use for the ethics analysis.

Result Analysis based on PLS. In order to analyze the feasibility and validation of ethics analysis based on PLS, 21variables are used as the input X of PLS. From the figure 1, we know the contribution of the 2th, 12th, 13th, 15th, 17th variables are obvious, especially the 2th, 13th and 15th variables. So, the 2th, 13th and 15th variables can be judged as common influence in portable intelligent devices. In the actual circumstances, the problem ethics for using portable intelligent devices are caused by the 2th, 13th and 15th, thus the partial derivatives contribution is very good for ethics analysis and illustrates the contribution degree based on partial derivatives in linear PLS is feasible in ethics analysis. 


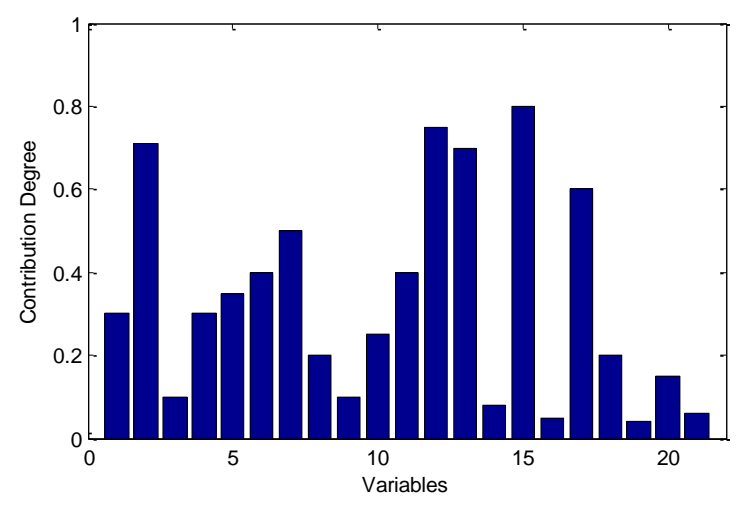

Figure 1. Variables contribution Chart

From figure 1, the effect of the model further demonstrates the feasibility of ethics analysis study, and establishes an effective discriminant analysis model. From the practical level, prediction of the model solves the ethics problem of portable intelligent devices by qualitative analysis. The model, in values, reveals that the biggest factors about impact of using portable intelligent devices are fatigue, security and privacy.

\section{Conclusion}

In this paper, we investigate the ethics problem for using portable intelligent devices and analyze the 21 variables about ethics. In order to find a model between human healthy and portable intelligent equipment, the PLS regression model is proposed to analyze the ethics problem about using portable intelligent devices. Through the data and result analysis, the proposed contribution degree reflects effectively the validation of some variables about ethics. The results show the impacts of fatigue, security and privacy are greater than other variables. Meantime, the model can explain ethics problem of portable intelligent devices at any time, so as to solve the vague diagnosis of the problem.

\section{Acknowledgment}

This research is supported by Humanities and Social Science Research Project in Colleges and Universities of Jiangxi Province(No: XL1406)

\section{Reference}

[1] J. D. Greene et al. An fMRI investigation of emotional engagement in moral judgment[J]. Science, 2001, 293:2105-2108.

[2] De Kang. Principle of moral metaphysics [M]. Shanghai: Shanghai people's press, 2002.

[3] D. Linden.The Compass of Pleasure[M].Viking Press, April 14,2011.

[4] B.Tal.Even Happier:A Gratitude Journal for Daily Joy and Lasting Fulfillment[M]. McGraw-Hill Professional, 2010.

[5] Ruobing Qian, Hongjun Guo. The present research situation and prospect of Internet addiction syndrome [J]. Chinese Journal of Stereotactic and Functional Neurosurgery, 2007, (1): 58-61.

[6] Yufang Bian. Revealing six big misunderstanding of the family education by brain science[R].China education newspaper, 2014, the seventh edition.

[7] Hyun-WooCho. Identieation of contribution variables using kernel-based discriminant modeling and reconstruction[J], ExPert-Systems with APPlieations, 2007.33:274-285. 
[8] Aziz Lazraq, Robert Cleroux, Jean-Pierre Gauchi. Selection both latent and explanatory variables in the PLS regression model, Chemommetrics and intelligent laboratory systems, 66, 2003:117-126 